\title{
Rosetta: Making History!
}

Revathi $\mathrm{B}^{1,{ }^{*}}$ and Prashanth $\mathrm{K}^{2}$

1Pharmaceutical Chemistry, Osmania University, Hyderabad, Telangana, India

2Mechanical Engineering, JNTU, Hyderabad, Telangana, India

"Corresponding author: Revathi B, M. Pharm, Pharmaceutical Chemistry, Osmania University, Hyderabad, Telangana, India, Tel: 91-9533484346; E-mail: revz.pharm@gmail.com

Received date: Nov 17, 2014, Accepted date: Nov 20, 2014, Published date: Nov 24, 2014

Copyright: (c) 2015 Revathi B. This is an open-access article distributed under the terms of the Creative Commons Attribution License, which permits unrestricted use, distribution, and reproduction in any medium, provided the original author and source are credited

\section{Abstract}

The first spacecraft to orbit a comet was Rosetta, which was launched by a European Space Operations Centre. Its aim is to study the comet 67P/Churyumov-Gerasimenko. The details of the orbiter and lander have been explained here.

Keywords: Rosetta; 67P; Churyumov-Gerasimenko; Orbiter; Philae; N.C. Wickramasinghe

\section{Overview of the Rosetta Mission}

Rosetta was the first spacecraft to orbit a comet. It was built and launched as a robotic space probe by European space agency European Space Operations Centre (ESOC), in Darmstadt, Germany. The aim of Rosetta is to study the comet with an orbiter and a lander module Phillae (Figure 1).

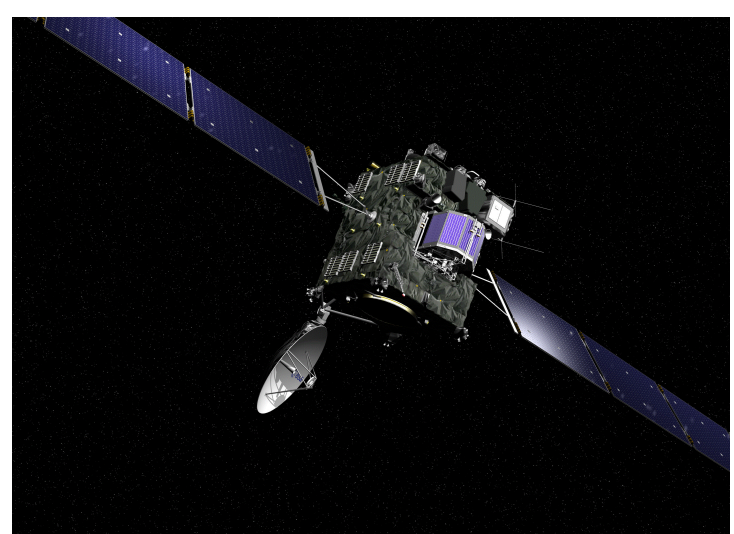

Figure 1: Rosetta

The comet is $67 \mathrm{P} /$ Churyumov-Gerasimenko. The launching of this spacecraft was done on March 2nd, 2004. It has reached the comet on August 6th, 2014. Rosetta is named after the Rosetta stone, an incomplete stela of black basalt incised with the same priestly decree in three scripts. Evidence for origin of life from comets has been growing steadily over the years. Sir Fred Hoyle and Dr. N.C. Wickramasinghe have contributed their research work on studying the comet and Rosetta mission is to provide a key deciphering the origin of life. It also aims in observing Mars during Mars Gravity Assist Maneuver. Also NASA has been involved with this mission. It has provided instruments to Rosetta [1-3].

\section{Previous Missions}

\section{International Cometry Explorer (ICE)}

Launched on August 12, 1978 built with a mission of studying the Earth's magnetic field and solar wind [4].

\section{Vega 1 and Vega 2}

These 2 Russian probes were launched on December 15 and 21, 1984 respectively. They were built with a mission to study the comet Halley. Each left a lander on the surface of Venus.

\section{Sakigake and Suisei}

These twin first Japans space missions were launched on 1985, 7 Jan and 18 Aug respectively. Its mission was to explore the comet Halley.

\section{Giotto}

Launched on 2 July, 1985, Europeans first mission. Giotto will continue to orbit the Sun for the foreseeable future, completing six revolutions roughly every seven years.

\section{Deep space 1}

This was the first spacecraft in NASA's New Millennium programme. Launched on 24 October 1998, its primary mission was to test 12 new advanced technologies.

\section{Stardust}

Launched on 7 Feb, 1999 is a mission led by NASA.

\section{Contour}

Launched on 3 July 2002, Contour was a NASA mission to improve our understanding of comet nuclei.

\section{Deep impact}

Another NASA's mission, launched on 12 Jan, 2005. 


\section{Rosetta}

Launched on 2 March, 2004.

\section{Technical Details of Rosetta}

Rosetta was built according to COSPAR rules. Rosetta consists of two elements, mainly; the Rosetta space probe orbiter that features 12 instruments, and the Philae robotic lander, which features 9 instruments. This spacecraft has been designed in such a way that it will orbit 67P for 17 months to complete the study of the comet [5].

\section{P/Churyumov-Gerasimenko Details}

Mass: $10^{13} \mathrm{~kg}$

Volume: $25 \mathrm{~km}^{3}$

Density: $0.4 \mathrm{~g} / \mathrm{cm}^{3}$

Rotation period: $12.404 \pm 0.0007^{*}$ hours

Orbital period: 6.55 years

Gases Detected: Water, $\mathrm{CO}, \mathrm{CO}_{2}, \mathrm{NH}_{3}, \mathrm{CH}_{4}, \mathrm{CH}_{3} \mathrm{OH}, \mathrm{Na}, \mathrm{Mg}$

Discovered by: Klim Churyumov and Gerasimenko

Year of Discovery: 1969

After its attachment to the comet, the lander was scheduled to commence its science mission:

- Characterization of the nucleus.

- Determination of the chemical compounds present, including amino acid enantiomers.

- Study of comet activities and developments over time [6].

\section{Timeline of the Mission as Programmed}

Launch of the mission: March 2004

Instrument checkout: April 2004

Earth gravity assist: March 2005

Deep impact observation: July 2005

Mars gravity assist: Feb 2007

Earth gravity assist: Nov 2007

Asteroid steins flyby: Sep 2008

Earth gravity assist: Nov 2009

Asteroid Lutetia flyby: Jul 2010

Enter Hibernation: July 2011

Exit Hibernation: Jan 2014

Rendezvous with the comet: May 2014

Global mapping of comet: Aug 2014

Philae landing on comet: Nov (12) 2014

Rosetta and comet reach perihelion (closest approach to sun): Aug 2015
End of mission: Dec 2015 [7,8].

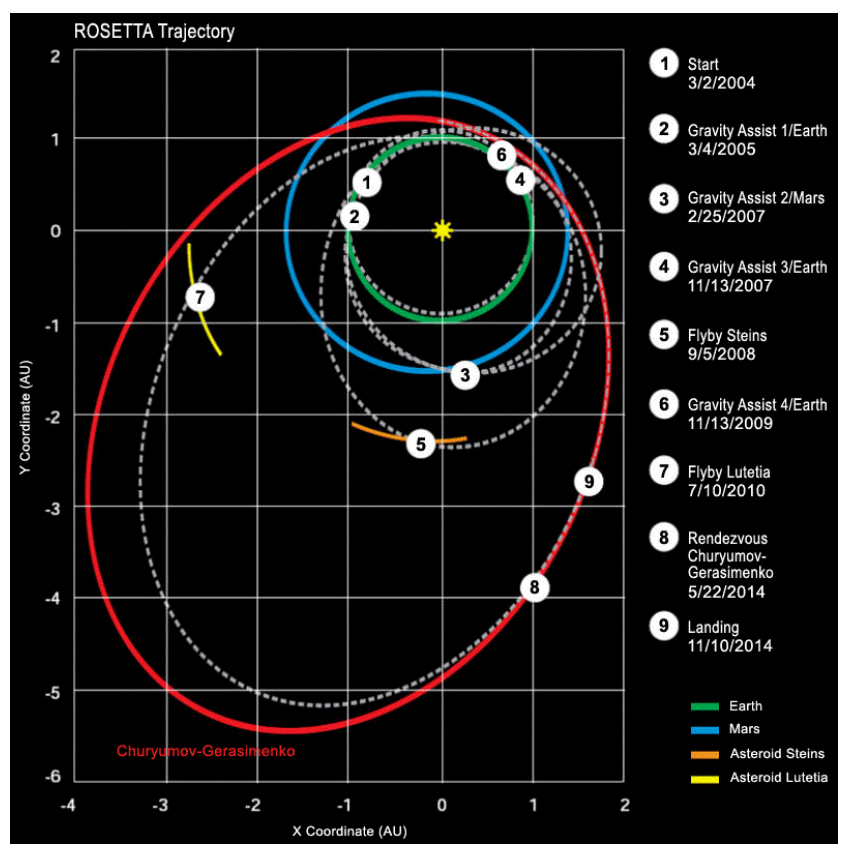

Figure 2: Programmed timeline of the Rosetta's mission

From all the above milestones, the mission has reached the stage where the lander Philae has landed on the comet successfully. This happened on Wednesday, November 12, 2014, the Rosetta mission landed successfully on the comets surface after three attempts. It landed at a speed of $2 \mathrm{mph}$. Philae; the lander detached and touched down first. By next August, i.e., 2015, the mission should reach perihelion and conduct its study (Figure 2).

The first touchdown was inside the predicted landing ellipse, confirmed using the lander's downwards-looking ROLIS descent camera in combination with the orbiter's OSIRIS images to match features $[7,8]$.

\section{References}

1. Fischer D (2014). Rendezvous with a crazy world. The Planetary Society.

2. Bauer M (2014) Rosetta Arrives at Comet Destination. European Space Agency.

3. Lakdawalla E (2014) Finding my way around comet ChuryumovGerasimenko. The Planetary Society.

4. Agl DC, Brown Dwayne, Bauer Markus (2014) Rosetta's Comet Target 'Releases' Plentiful Water European Space Agency. NASA.

5. Chang, Kenneth (2014) Rosetta Spacecraft Set for Unprecedented Close Study of a Comet. New York Times.

6. Meierhenrich, Uwe (2008) Amino Acids and the Asymmetry of Life. Springer-Verlag.

7. Jet propulsion laboratory (2014) California Institute of Technology. NASA.

8. Mottola S (2014) The rotation state of 67P/Churyumov-Gerasimenko from approach observations with the OSIRIS cameras on Rosetta. Astronomy \& Astrophysics 569: L2. 